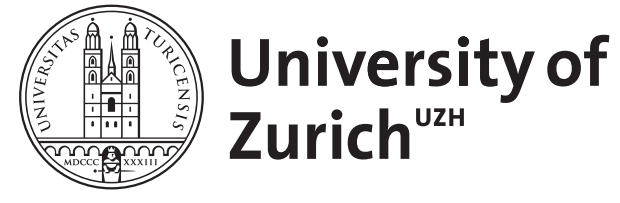

Zurich Open Repository and Archive

University of Zurich

University Library

Strickhofstrasse 39

CH-8057 Zurich

www.zora.uzh.ch

Year: 2014

\title{
Literarische Bildlichkeit und Rhetorik
}

Berndt, Frauke

Posted at the Zurich Open Repository and Archive, University of Zurich

ZORA URL: https://doi.org/10.5167/uzh-125072

Book Section

Published Version

Originally published at:

Berndt, Frauke (2014). Literarische Bildlichkeit und Rhetorik. In: Benthien, Claudia; Weingart, Brigitte. Handbuch Literatur Visuelle Kultur. Berlin: De Gruyter, 48-67. 


\section{Handbuch Literatur \& Visuelle Kultur}

Herausgegeben von

Claudia Benthien und Brigitte Weingart

\section{DE GRUYTER}


ISBN 978-3-11-028565-9

e-ISBN [PDF] 978-3-11-028576-5

e-ISBN [EPUB] 978-3-11-038165-8

ISSN 2197-1692

\section{Library of Congress Cataloging-in-Publication Data}

A CIP catalog record for this book has been applied for at the Library of Congress.

\section{Bibliografische Information der Deutschen Nationalbibliothek}

Die Deutsche Nationalbibliothek verzeichnet diese Publikation in der Deutschen Nationalbibliografie; detaillierte bibliografische Angaben sind im Internet über http://dnb.dnb.de abrufbar.

(C) 2014 Walter de Gruyter GmbH, Berlin/Boston

Satz: fidus Publikations-Service GmbH, Nördlingen

Druck und Bindung: Hubert \& Co. GmbH \& Co. KG, Göttingen

@ Gedruckt auf säurefreiem Papier

Printed in Germany

www.degruyter.com 


\section{Inhaltsverzeichnis}

1. Einleitung - Claudia Benthien und Brigitte Weingart — 1

2. Theoretische Perspektiven

2.1 Visualität und Materialität der Literatur - Stephan Kammer -31

2.2 Literarische Bildlichkeit und Rhetorik - Frauke Berndt -48

2.3 Die Laokoon-Debatte: Kunstreflexion und Medienkonkurrenz im 18. Jahrhundert - Sabine Schneider -68

2.4 Bilder-Denken und Symbolisierungsprozesse in der frühen Kulturwissenschaft - Barbara Naumann — 86

2.5 Literarische Lektüre und imaginative Visualisierung: Kognitionsnarratologische Aspekte - Renate Brosch — 104

2.6 Semiologie und Rhetorik des Bildes - Petra Löffler — 121

2.7 Intermedialität: Text/Bild-Verhältnisse - Gabriele Rippl 139

2.8 Visual Culture - Bernd Stiegler 159

3. Problematisierungen und Forschungsfragen

3.1 Der blinde Fleck der Disziplinen: Zwischen Bild- und Textwissenschaften - Michael Wetzel 175

3.2 Von der Semiologie zur ,visuellen Literalität‘? Isabel Capeloa Gil — 193

3.3 Ausnahmesituationen: Verhandlungen der Darstellbarkeit von existentiellem Leid - Silke Segler-Meßner — 212

3.4 Pornografie und Bildkritik in Texten des 20. Jahrhunderts Peter Rehberg — 229

3.5 Schreibweisen der Oberfläche und visuelle Kultur Heinz Drügh — 247

3.6 Literarizität in der Medienkunst - Claudia Benthien — 265 
4. Medienhistorische Konstellationen und exemplarische Analysen

4.1 Ekphrasis in der Literatur des Mittelalters - Haiko Wandhoff — 287

4.2 Visualität in der höfischen Literatur und Kultur des Mittelalters Hans Rudolf Velten $\mathbf{3 0 4}$

4.3 Textualität und Visualität um 1500 - Helmut Puff- 321

4.4 Sinn-Bilder': Emblematik in der Frühen Neuzeit Wolfgang Neuber — 341

4.5 Augenzeugenschaft und sprachliche Visualisierung im Drama (A. Gryphius: Catharina von Georgien, H. von Kleist: Penthesilea) Claudia Benthien — 357

4.6 Literatur als Reflexionsmedium von Visualität: Mediologische Perspektiven auf das Panorama - Ralph Köhnen — 375

4.7 Effekte des Realen: Bildmedien und Literatur im Realismus (G. Flaubert: L'Éducation sentimentale) - Barbara Vinken — 393

4.8 Foto-Poetiken der Erinnerung in der Literatur des 20. Jahrhunderts (M. Proust: À la recherche du temps perdu) - Irene Albers — 408

4.9 Grenzen in Bewegung: Bild und Text in den historischen Avantgarden - Nicola Behrmann — 426

4.10 ,Kinematografie“: Filmische Schreibweisen in der Literatur der Weimarer Republik - Stefanie Harris — 445

4.11 Visualisierung und Narrativierung in Erzähltexten der Moderne (H. Green: Blindness) - Ronja Tripp — 462

4.12 Autor/innen-Porträts: Vom Bildnis zum Image Matthias Bickenbach — 478

4.13 Fotobücher: Bildserien in Bänden - Christoph Ribbat $\mathbf{- 5 0 0}$

4.14 „Sehtextkommentar“: Schriftbilder, Bilderschriften (F. Kriwet) Brigitte Weingart — 519

4.15 Grafische Literatur (A. Bechdel: Fun Home und D. Small: Stitches) Astrid Böger — 544

5. Glossar - Claudia Benthien und Brigitte Weingart — $\mathbf{5 6 1}$ 
6. Auswahlbibliografie -593

7. Register -613

7.1 Personenregister -613

$7.2 \quad$ Sachregister -627

8. Abbildungsnachweise -637

9. Autorinnen und Autoren -641 


\title{
2.2 Literarische Bildlichkeit und Rhetorik
}

\author{
Frauke Berndt
}

\section{Sprachbildlichkeit}

Ut pictura poesis („Das Dichtwerk gleicht dem Gemälde“) steht in den Lehrbüchern der antiken Poetik und ausdrücklich in dieser Wendung bei Horaz geschrieben (vgl. Horaz 1967 [ca. 14 v. Chr.], 250-251). Dass literarische Texte freilich ihre eigene, medienspezifische Bildlichkeit haben, führt von der Poetik zur Rhetorik zurück. Ut pictura oratio steht zwar in keinem Lehrbuch der antiken Rhetorik, dennoch: Ebenso wie gute Bilder der Dichtung zur Schönheit verhelfen, verschaffen sie dem Redner - Rednerinnen sind in dieser historischen Disziplin nicht oder nur am Rand vorgesehen (vgl. Tonger-Erk 2012) - vor Gericht oder auf dem Marktplatz Gehör. Und weil sie die sprachlichen Mittel der Bildlichkeit und deren Wirkung nicht nur genau beobachtet, sondern auch auf Begriffe bringt und in einem System integriert, bildet die Rhetorik die Grundlage jeder literarischen Medienästhetik, die bis heute sowohl die allgemeinen Stilkategorien als auch die besonderen Namen der sprachlichen Gestaltungsmittel verwendet. Wolfram Groddeck verhandelt die Rhetorik deshalb nicht von ungefähr als Stilistik des Lesens, weil ihr begriffliches Wissen der Analyse literarischer Texte gute Dienste leistet - insbesondere der Analyse ihrer Bildlichkeit (vgl. Groddeck 2008 [1995]).

Die Konjunktion ut (,wie') im Appell an die Dichtung macht schon deutlich, dass der Ausdruck ,literarische Bildlichkeit' selbst ein bildlicher Ausdruck ist. Denn wie kann ein literarischer Text, der sich sprachlicher Zeichen bedient, bildlich sein? Doch wohl nur dadurch, dass er etwas zu leisten im Stande ist, das sich am Funktions- und Leistungsprofil des Bildes bemisst, sodass das Bild zu seinem Referenzmedium wird. Mit dieser Referenz wird freilich die Unterscheidung zwischen Vorstellungen, Künsten und Medien verwischt: Als Bild gelten sowohl mentale als auch konkrete Bilder - hier wiederum alle Bilder vom Piktogramm bis zum Gemälde. Die Eigenschaft der Bildlichkeit kann Gegenständen nämlich dann zugeschrieben werden, wenn sie eine bestimmte kognitive Wirkung haben - die Wirkung, anschauliche Vorstellungen zu erzeugen (vgl. Willems 1989). Obwohl es sich also bei Sprache und Bild um unterschiedliche Modalitäten handelt, weil sie verschiedenen Zeichensystemen entsprechen, kann beiden die Eigenschaft der Bildlichkeit zugeschrieben werden. In diesem Sinn ist ,Sprachbildlichkeit' also die Eigenschaft der Sprache, anschauliche Vorstellungen zu erzeugen (vgl. 2.5 BROSCH). Dafür verwendet die Sprache freilich andere Techniken als das wahrnehmungsnahe Medium des Bildes (vgl. Sachs-Hombach 2013 [2003]). Diese Ver- 
fahren „rhetorischer Bildgebung“ (Campe 1997, 208) werden in der Rhetorik auf eine Art und Weise reflektiert, die bis heute aktuell ist. Denn als Medientheoretiker avant la lettre loten die Rhetoriker die Möglichkeiten der Sprache und ihrer medialen Realisierungen in mündlicher Rede und schriftlichem Text aus, bildlich $\mathrm{zu}$ verfahren.

In der Summe zielt die antike Rhetorik auf die umfassende Ausbildung des Redners zu einem nicht nur sprachgewandten, sondern auch zu einem ethisch ,guten Mann' (vir bonus) ab. Deshalb umfassen die Lehrbücher neben dem sprachlichen Regelwerk auch didaktische, ethische, pädagogische und politische Aspekte. (Er-)Findungslehre (inventio), Anleitungen zur Ordnung der Rede (dispositio), Darstellungs- beziehungsweise Stillehre (elocutio), Gedächtnislehre (memoria) sowie Anleitungen zum Aufführen der Rede (actio) bilden die fünf Teile des in der Ausführung dann variablen und flexiblen rhetorischen Systems (officia oratoris). Für die bildgebenden Verfahren ist die Stillehre (elocutio) zuständig, die im Wesentlichen vier Probleme behandelt und dementsprechend fünf Stilkategorien vorgibt: Kürze (brevitas) und Klarheit (perspicuitas) der Rede, Richtigkeit (Orthografie und Grammatik) der Sprache (puritas), Redeschmuck (ornatus) sowie Angemessenheit des Ausdrucks im jeweiligen Redekontext (aptum). Historisch wird das System bis ins 18. Jahrhundert überliefert (vgl. Campe 1990). Seitdem übernimmt die Stillehre neue Funktionen in der literarästhetischen Medientheorie (vgl. Berndt 2011).

In der Stillehre katalogisiert der römische Redner Quintilian in seiner Institutio oratoria (95 n. Chr.; Ausbildung des Redners) den Redeschmuck, den er grundsätzlich in Figuren und Tropen unterteilt. Als Figuren bezeichnet er alle Abweichungen vom ,normalen', eben schmucklosen Sprachgebrauch: das Weglassen, Hinzufügen und Umstellen von Wörtern, Satzteilen und Sätzen, sowie darüber hinaus den performativen Gebrauch von Äußerungen, den die Gedankenfiguren (figurae sententiarum) abdecken - Fragen, Zweifeln, Behaupten, Appellieren etc. Quintilian geht davon aus, dass sich bei den Figuren die Bedeutung der Wörter nicht verändert, die durch das Wörterbuch bestimmt wird. Bei den Tropen deshalb werden sie ,Sprachbilder' genannt - hingegen ändert sich die Bedeutung der Wörter. Sowohl Figuren als auch Tropen tragen zur Bildlichkeit der Rede bei.

\section{Veranschaulichen und Verlebendigen}

Vor der Aufgabe, einem Gegenstand Klarheit zu verleihen, steht jeder Orator. Mit der Definition der dafür zuständigen Gedankenfigur reiht sich Quintilian in die römische Tradition ein. Er beruft sich auf Ciceros Abhandlungen De oratore 
(55 v. Chr.; ,Über den Redner') sowie Orator (46 v. Chr.; ,Der Redner`), in denen

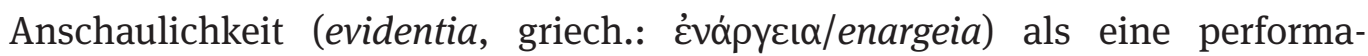
tive Figur definiert wird (vgl. Cicero 1998 [46 v. Chr.], 119, und 1976 [55 v. Chr.], 573-575): „Die Figur nun, die Cicero als Unmittelbar-vor-Augen-Stellen bezeichnet, pflegt dann einzutreten, wenn ein Vorgang nicht als geschehen angegeben, sondern so, wie er geschehen ist, vorgeführt wird, und nicht im Ganzen, sondern in seinen Abschnitten“ (Quintilian 1995b [ca. 95 n. Chr.], 287). Die Unterscheidung, auf die es Quintilian bei seiner Definition ankommt, ist diejenige zwischen propositionalem Sagen (indicare) und theatralem Zeigen (ostendere). Anschaulichkeit steht also weniger im Dienst der Repräsentation von Vergangenem - ,als geschehen“ -, sondern im Dienst der Aktualisierung, die auf einer der ursprünglichen Situation analogen Präsenz - „wie es geschehen ist“ - basiert: Der römische Enzyklopädist Aulus Cornelius Celsus, den Quintilian nun anführt, „hat auch die Figur der Aktualisierung selbst ,Anschaulichkeit' [...] benannt, bei

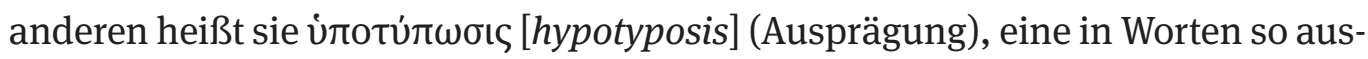
geprägte Gestaltung von Vorgängen, daß man eher glaubt, sie zu sehen als zu hören“ (ebd.) (siehe zu diesem Zusammenhang auch 4.5 BENTHIEN).

Im Spannungsfeld von Psychologie und Medienästhetik bestimmt Quintilians Definition die emotionale Wirksamkeit der Rede an der Vergegenwärtigung der Gegenstände. Im Gegensatz zum Bild besteht bei der Rede indes keine Ähnlichkeit zwischen dem signifikanten Material (Zeichenträger) und dem Referenten. Die Bildlichkeit der Rede findet vielmehr ,im Kopf‘ der Hörerinnen und Hörer statt, ja verdankt sich einem Akt der (Vor-)Täuschung. Wenn aber unter dieser Voraussetzung von einer Rede beziehungsweise - auf diesen Transfer soll es hinauslaufen - von einem literarischen Text gefordert wird, dieselbe Wirkung wie ein Bild zu erzeugen, dann steht für Quintilian dasselbe Problem zur Lösung an, dem sich später Gotthold Ephraim Lessing gewidmet hat: Nicht im Ganzen (nec universa), überlegt Quintilian, kann Sprache einen sich zeitlich ausdehnenden Vorgang (res gesta) simultan darstellen, sondern in seinen Abschnitten (per partis). In der sprachlichen Sukzessivität entsteht also die Pseudo-Simultaneität der Anschaulichkeit. Deshalb betont Quintilian an den sprachlichen Verfahren der Veranschaulichung ausdrücklich die Selbstreferenz gegenüber der Fremdreferenz, oder anders gewendet (vgl. ebd.): Er gibt der Semiosis gegenüber der Mimesis den Vorrang (vgl. Riffaterre 1978b), „weil die Veranschaulichung (evidentia) oder, wie andere sagen, Vergegenwärtigung (repraesentatio) mehr ist als die Durchsichtigkeit (perspicuitas), weil nämlich letztere nur den Durchblick gestattet (patere), während die erstere sich gewissermaßen selbst zur Schau stellt (ostendere)“(Quintilian 1995b [ca. 95 n. Chr.], 177).

In der „Fülle seiner Rollen und der Vielfalt in Ersetzungsart und Wirkung erscheint das Vor-Augen-Stellen ,selbst‘ wie die Unbekannte einer Funktion“, so 
erläutert Rüdiger Campe die rhetorischen Suchbewegungen (Campe 1997, 209) die Unbekannte einer Funktion ,Bildlichkeit‘, bei deren performativer Bestimmung Quintilian nicht nur mit Celsus und Cicero, sondern vor allem auch mit Aristoteles rechnet. In dessen Rhetorik (ca. 349-336 v. Chr.) heißt es: „Es muss

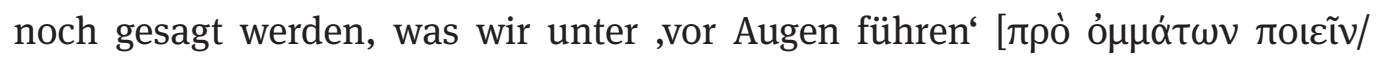
pro ommaton poiein] verstehen und was man tun muss, damit dies | zustande kommt. Ich sage nämlich von allem dem, dass es vor Augen führt, was etwas in einer Aktivität Befindliches bezeichnet“, wobei Aristoteles den Akzent nicht auf die Veranschaulichung ('́vópycı//enargeia), sondern auf die Verlebendigung ('́vépycı/energeia) legt: „[D]ie Aktivität ist aber eine Bewegung“ (Aristoteles 2002 [ca. 349-336 v. Chr.], 146-147). Veranschaulichung und Verlebendigung sind in ihrer langen Begriffsgeschichte sowohl in Konzepten der Ergänzung als auch des Ersatzes stets aufeinander bezogen worden. Diese zum Teil „innerterminologische Verwechslung“ geht auf die Rechnung einer „eklektische[n] Zusammenschau verschiedener Sprachkonzepte“ (Campe 1990, 230, Anm. 22) - der Zusammenschau des visuell-repräsentativen Sprachkonzepts der römischen Spätantike mit einem auf Aristoteles zurückgehenden energetisch-performativen Sprachkonzept, dessen Spuren in Quintilians selbstreferentieller Definition der Veranschaulichung noch zu finden sind. Campe geht sogar so weit, die Gedankenfigur des Vor-Augen-Stellens als „Metafigur der Rhetorik“ zu bezeichnen (ebd., 209).

Während Quintilian buchstäblich auf die Inszenierung von Gegenständen abhebt und die Funktion ,Bildlichkeit' im Horizont des Vergangenen denkt, bestimmt Aristoteles die Funktion als Verlebendigung unbelebter Gegenstände im Horizont der Zukunft. Die Beispiele in der aristotelischen Rhetorik stammen nicht von ungefähr aus literarischen Texten. Über Homer, dessen Epen Aristoteles für besonders gelungen hält, äußert er sich folgendermaßen: „[B]ewegt und lebendig macht er nämlich alles, die Aktivität ist aber eine Bewegung“ (Aristoteles 2002 [ca. 349-336 v. Chr.], 147). Als Referenzmedium dient Aristoteles daher auch nicht das Bild; vielmehr greift er auf ein physikalisches Modell zurück. Mit der Bewegung geht es ihm um das Prinzip der Verwirklichung einer Möglichkeit.

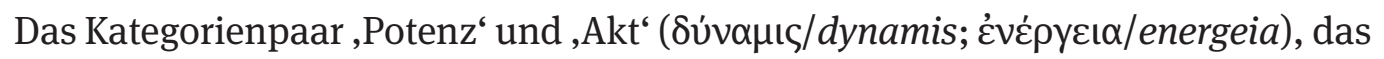
dieses Prinzip ausdrückt, hat Aristoteles bereits aus der Physik in die Metaphysik und in die Nikomachische Ethik (alle 4. Jh. v. Chr.) übersetzt. In Rhetorik und Poetik (beide um 335 v. Chr.) bindet er die Bewegung nun sowohl an unbildliche Verfahren der Aktualisierung (Verbformen des Präsens, Imperativ, Apostrophe, direkte Rede) als auch an bildgebende Verfahren. Neben der Metonymie, die „Unbeseeltes [...] zu Beseeltem“ macht, spielt vor allem die „nach der Analogie gebildete[] Metapher“ eine zentrale Rolle für die Verlebendigung (ebd., 146). Mit ihrem Bewegungsaspekt dynamisiert Aristoteles die statische Funktion ,Bildlichkeit‘, sodass in der Folge die emotionale Wirksamkeit der Rede gegenüber der 
Veranschaulichung gesteigert wird. Diesem Umstand trägt auch Quintilian in seiner Affektenlehre Rechnung, in der er die Gefühlswirkungen der „Phantasiebilder“ (visiones) analysiert: „Daraus ergibt sich die ćvópycı $\alpha$ [enargeia] (Verdeutlichung), die Cicero illustratio (Ins-Licht-Rücken) und evidentia (Anschaulichkeit) nennt, die nicht mehr in erster Linie zu reden, sondern vielmehr das Geschehene anschaulich vorzuführen scheint, und ihr folgen die Gefühlswirkungen so, als wären wir bei den Vorgängen selbst zugegen“ (Quintilian 1995a [ca. 95 n. Chr.], 711).

\section{Vergegenwärtigen}

Die Spannung zwischen Fremd- und Selbstreferenz der Anschaulichkeit tritt auch

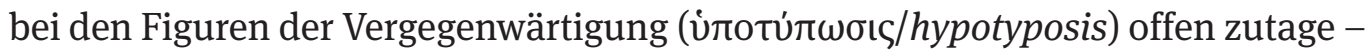
das sind vor allem: Metapher ( $\mu \varepsilon \tau \alpha \varphi \circ \rho \alpha$ /metaphora), Metonymie ( $\mu \varepsilon \tau \omega \nu \mu \mu i ́ \alpha /$

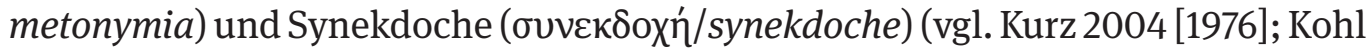
2007). Ebenso wenig wie bei den beiden Gedankenfiguren geht es bei den Tropen lediglich um bildgebende Verfahren im Besonderen. Denn eigentlich interessiert die Rhetorik das Funktions- und Leistungsprofil symbolischer Zeichen (signa visibiles) im Allgemeinen (vgl. Berndt 2011). Die Formen der Vergegenwärtigung sind dementsprechend vielfältig: Bildlich werden vor allem Verben, Adjektive und Nomen - hier vor allem Konkreta und Eigennamen - in unterschiedlichen grammatischen Funktionen verwendet. Deren durch ihren Gegenstandsbezug ohnehin bereits gegebene Anschaulichkeit wird durch eine von Theorie zu Theorie ganz unterschiedlich begründete Bedeutungserweiterung gesteigert. Vor allem der aristotelischen Semantik liegt daher eine voraussetzungsreiche Ontologie zugrunde (vgl. Ricœur 1986 [1975]), wenn er definiert: „Denn gute Metaphern zu finden, hängt von der Fähigkeit ab, Ähnlichkeiten [d.h. in Verschiedenem das Gleiche] zu erkennen.“ (Aristoteles 2011 [ca. 335 v. Chr.], 33) Unter den drei Metapherntypen rangiert bei Aristoteles die Analogiemetapher an oberster Stelle: „Von einer analogen ,Verwendungsweise“ spreche ich, wenn sich das Zweite zum Ersten genauso verhält wie das Vierte zum Dritten. Man wird nämlich anstelle des Zweiten das Vierte oder anstelle des Vierten das Zweite nennen. Und manchmal setzt man hinzu, | worauf der Ausdruck, der stellvertretend verwendet wird, bezogen ist“ (ebd., 30). Die Proportionen folgen dem Schema des Dreisatzes (vgl. Groddeck 2008 [1995], 257): A : B = C : D. Über Kreuz erhält man die folgende Gleichung: $\mathrm{A}: \mathrm{D}=\mathrm{C}: \mathrm{B}$.

In seinen Beiträgen zu einer Kritik der Sprache (1912) erklärt Fritz Mauthner die aristotelische Tropologie folgendermaßen: „Es verhalte sich z. B. die Trink- 
schale des Dionysos zu diesem Gotte wie der Schild zum Gotte Ares“ (Mauthner 1967 [1912], 457). Die Pointe besteht darin, dass man „darum ganz mechanisch die Glieder der Proportion miteinander vertauschen und geistreich sagen“ könne, „die Trinkschale sei der Schild des Dionysos (was immerhin nicht ohne Witz wäre, füge ich hinzu), oder der Schild sei die Trinkschale des Ares (was schon recht abgeschmackt wäre)“ (ebd.). Offenbar stört sich Mauthner daran, dass diese Metapher den Gott des Blutbades und Massakers zu einem Vampir macht. Nicht


katachresis), bei der ein lexikalisches Element fehlt, funktioniert das AnalogieVerfahren tadellos, zum Beispiel bei der Metapher ,Säend das göttliche Licht‘. Die Tätigkeit der Sonne (A) verhält sich zum göttlichen Licht (B) wie das Säen (C) zum Samen (D). Über Kreuz erhält man die Metapher für das fehlende Prädikat zum Subjekt des Sonnenlichts.

Im Spannungsfeld von Fremd- und Selbstreferenz zeigen aber auch die aristotelischen Beispiele, dass es konzeptuell zu kurz greift, literarische Bildlichkeit auf Anschaulichkeit und Anschaulichkeit auf Gegenständlichkeit zu verpflichten. Die auf Aristoteles folgenden Definitionen entkoppeln die Metapher deshalb nicht von ungefähr von den ontologischen Grundlagen. Dementsprechend wertet Quintilian die Tropik zur universalen Übersetzungsmaschine auf beziehungsweise um: „Ein Tropus ist die kunstvolle Vertauschung der eigentlichen Bedeutung eines Wortes oder Ausdruckes mit einer anderen“ (Quintilian 1995b [ca. 95 n. Chr.], 217). Tropen operieren hier bloß noch auf der Ebene des Zeichens: „Übertragen wird also ein Nomen oder Verbum von der Stelle, wo seine eigentliche Bedeutung liegt, auf die, wo eine eigentliche (proprium) Bedeutung fehlt oder die übertragene (improprium) besser ist als die eigentliche“ (ebd., 219). Jacques Derrida erkennt in der Metapher daher die strukturelle Matrix aller „symbolischen oder analogen Figuren“, die er per definitionem für selbstreferentiell hält: „Die Metapher wurde immer definiert als die Trope der Ähnlichkeit; nicht bloß zwischen einem Signifikant und Signifikat, sondern bereits zwischen zwei Zeichen, von denen das eine das andere darstellt“ (Derrida 1988 [1972], 210). Als Übersetzungen (translationes) dienen die Metapher und sämtliche ihr nebenoder untergeordneten Tropen der Bedeutungsverschiebung (synecdoche) und Umbenennung (metonymia) stets der Ersetzung von Abstrakta durch Konkreta, damit die Anschaulichkeit des literarischen Textes zunimmt. Davon zeugt nicht zuletzt die schlichte Definition der Metapher als verkürzter Vergleich (metaphora brevior est similitudo): „Eine Vergleichung (comparatio) ist es, wenn ich sage, ein Mann habe etwas getan ,wie ein Löwe‘, eine Metapher, wenn ich von dem Mann sage: ,er ist ein Löwe““ (Quintilian 1995b [ca. 95 n. Chr.], 221). 


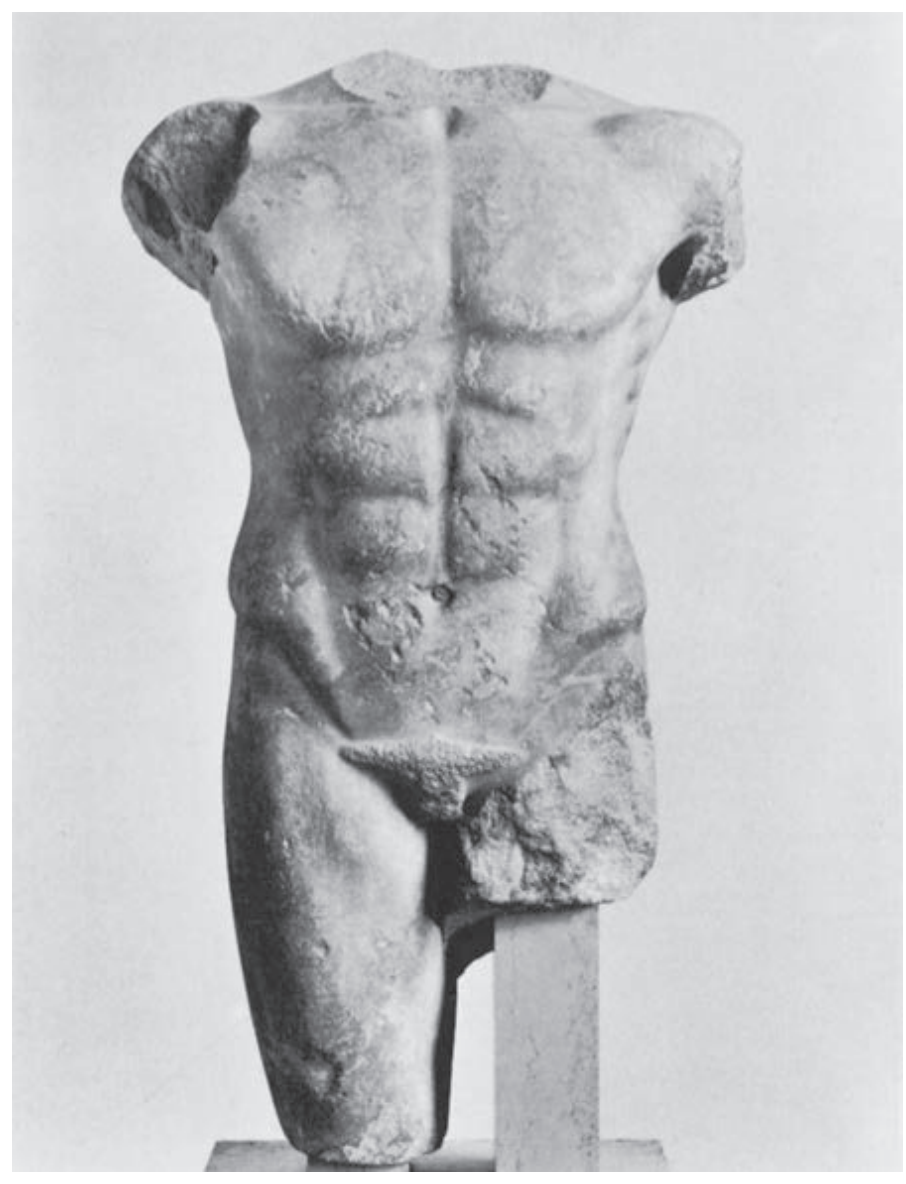

Abb. 1: Torso aus Milet, Marmorplastik, um oder nach 480 v. Chr.

Wie Fremd- und Selbstreferenz bei den Tropen ineinandergreifen, zeigt die literarische Bildlichkeit in Rainer Maria Rilkes Sonett auf den Torso von Milet (Abb.1). Im Archaïschen Torso Apollos (1908) ersetzt Rilke die Wahrnehmung des entstellten Steins durch die Imagination eines lächelnden Gesichts:

Archaïscher Torso Apollos

Wir kannten nicht sein unerhörtes Haupt, darin die Augenäpfel reiften. Aber sein Torso glüht noch wie ein Kandelaber, in dem sein Schauen, nur zurückgeschraubt,

sich hält und glänzt. Sonst könnte nicht der Bug der Brust dich blenden, und im leisen Drehen der Lenden könnte nicht ein Lächeln gehen zu jener Mitte, die die Zeugung trug. 


\begin{abstract}
Sonst stünde dieser Stein entstellt und kurz unter der Schultern durchsichtigem Sturz und flimmerte nicht so wie Raubtierfelle;

und bräche nicht aus allen seinen Rändern aus wie ein Stern: denn da ist keine Stelle, die dich nicht sieht. Du mußt dein Leben ändern.
\end{abstract}

(Rilke 1996 [1908], 513)

Den Ausgangspunkt dieses Prozesses bildet eine Negation („wir kannten nicht“), die den metaphorischen Prozess in Gang setzt: Der Torso ist buchstäblich kopflos. Dass das unerhörte Haupt eine Synekdoche für den griechischen Gott des Lichts, der Weissagung und der Künste (vor allem der Musik, aber auch der Dichtung) ist, wird vom Schluss des Sonetts her deutlich, an dem dieser Gott spricht; nach 13 Versen des Sonetts wird er im letzten nun endlich ,erhört'. Doch gehorcht die auf die Negation folgende Imagination keinen mimetischen Regeln. Bereits der erste Anschluss im zweiten Vers mit dem Pronominaladverb ,darin' ist ebenso ungrammatisch wie der folgende Ausdruck der reifenden Augenäpfel. Metaphorisch wäre er, würde man das Verb ,reifen' im Kompositum auf das Genitivattribut ,Augen beziehen; im Bezug auf die Äpfel ist das Reifen aber keineswegs metaphorisch. Die semantische Doppeldeutigkeit (ambiguitas) des Ausdrucks sorgt insofern für Unanschaulichkeit, als sie gegen die Stilkategorie der Klarheit verstößt. Denn

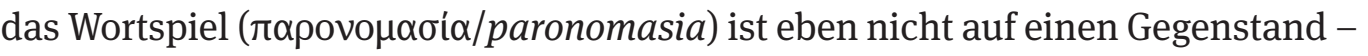
weder die Augen noch die Äpfel - hin durchsichtig, sondern erzeugt sprachliche Undurchsichtigkeit (obscuritas). Diese Opazität nimmt mit der komplexen Bildlichkeit noch weiter zu, die durch die adversative Verbindung des Hauptes mit dem Körper gesteigert wird. Dabei wird der Körper mit einer Leuchte verglichen: „Aber sein Torso glüht noch wie ein Kandelaber“. Im Relativsatz anagrammiert Rilke das Wortfeld des Sinnesorgans ,Auge' mit dem Wortfeld der Leuchttechnik des Kandelabers (vgl. Starobinski 1980, 125-126).

Was in Rilkes Sonett der Macht der philologischen Gewohnheit folgend als literarische Bildlichkeit bezeichnet wird, verdankt sich bei genauerer Überlegung einer topischen Kombinatorik. Denn die vermeintlichen Bilder sind Topoi, das heißt semantische Versatzstücke aus dem Apollon-Archiv, sodass man Rilkes bildgebendes Verfahren als etymologisches bezeichnen könnte (vgl. Willer 2004, 39). Alle visuellen Ausdrücke, die organischen wie die technischen, verweisen auf die Licht- oder Feuernatur des Gottes, die „Raubtierfelle“ auf seine Löwengestalt. Die Etymologie führt vom Glänzenden über die Sonne zum ,Äugler‘; ja selbst der Apfelgott Apollon kann im Archiv gefunden werden (vgl. Fauth 1964, 441). Dass die Bilder im Irrealis stehen, betont das Paradox anschaulicher Unan- 
schaulichkeit, das wie gehabt durch die Spannung zwischen Fremd- und Selbstreferenz entsteht. Jedenfalls erzeugen die Verse eine dergestalt surrealistische Gegenständlichkeit wie sie René Magrittes Gemälde Le viol (,Die Vergewaltigung; 1934) abbildet (Abb.2). ,Vergewaltigt“ oder zumindest: missverstanden wird Rilkes Sonett nämlich, sobald der Rezipient die einzelnen Bilder ,in seinem Kopf ${ }^{\star}$ hermeneutisch zu einem Ganzen zusammensetzt. Gegenüber seinen Teilen verliert ein solches Ganzes sowohl an semantischer Komplexität als auch an emotionaler Wirksamkeit.

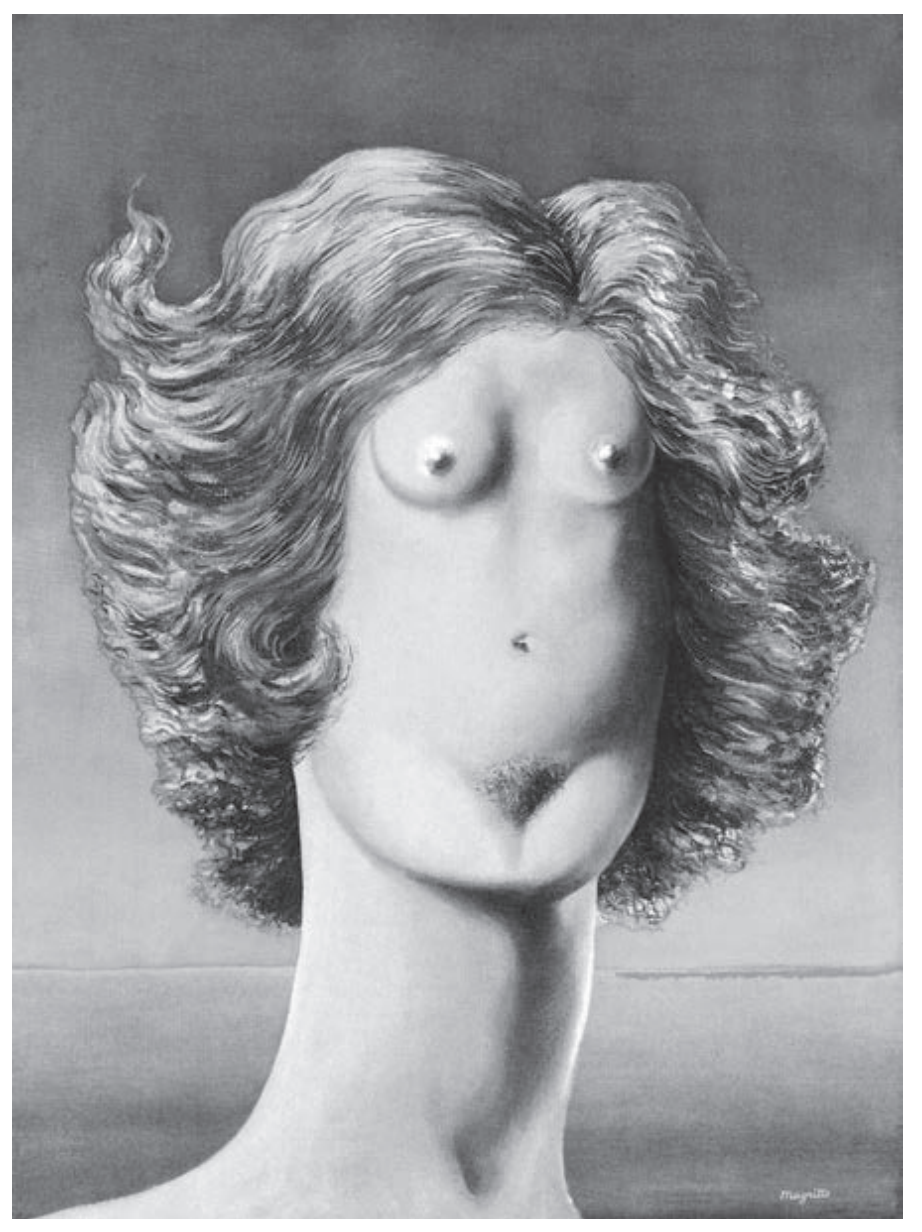

Abb. 2: René Magritte: Le viol, Öl auf Leinwand, 1934

Mit dem Paradox anschaulicher Unanschaulichkeit setzt Rilkes Sonett offenbar weniger auf statische Veranschaulichung in quintilianscher Tradition als auf dynamische Verlebendigung in aristotelischer Tradition, weniger auf Repräsentation als auf Präsenz. Sie wird nicht zuletzt dadurch erreicht, dass die einzelnen Teile des ,Haupt-Körpers“ durch Verbmetaphern aktiviert werden: reifen, glühen, glänzen, blenden, flimmern, ausbrechen. Da Aristoteles nur beseelten Gegenständen Strebungsvermögen (Willen) zuspricht, ist es die Aufgabe der Metapher, 
unbeseelte Gegenstände zu beseelen. In Homers Ilias findet Aristoteles etwa das Beispiel: „Und die Spitze fuhr ihm durch die Brust hindurch, begierig“ (Homer 1977 [8./7. Jh. v. Chr.], 257; vgl. Aristoteles 2002 [ca. 349-336 v. Chr.], 147). Um die Brust des Feindes zu durchbohren, stürmt der Pfeil dahin. Sein eigentliches

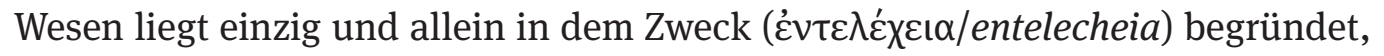
den Feind zu töten.

In den römischen Lehrbüchern werden dergestalt emotionale ,Sprachbilder nicht in der Stillehre, sondern in den Gedächtnislehren behandelt. In diesem Teil werden alle Schwierigkeiten, Probleme, Aporien und Ambiguitäten der Rhetorik in das System zurückgeholt, sodass man ihn als einen re-entry innerhalb des rhetorischen Systems bezeichnen kann (vgl. Berndt 2004, 33-35). In ihrem Zentrum steht das theatrale Bild (imago agens), das eben gerade kein mimetisches Bild ist. Genauso wie Rilkes Metaphern sind die imagines agentes emotionale und dem Gedächtnis deshalb gut einprägsame Bilder, die nicht fremd-, sondern selbstreferentiell konzipiert sind, das heißt, denen ,keine bildliche Analogie (nulla similitudine)“ zugrunde liegt (Cicero 1976 [55 v. Chr.], 437). In der von einem Unbekannten im ersten vorchristlichen Jahrhundert verfassten Rhetorik De ratione dicendi ad C. Herennium (ca. 86-82 v. Chr.) heißt es in diesem Sinn: „Da nun also den Vorgängen die Bilder ähnlich sein müssen und wir aus allen Vorgängen für uns Ähnlichkeiten auswählen müssen, müssen also die Ähnlichkeiten doppelt sein, zum einen mit den Vorgängen, zum anderen mit den Worten (unae rerum, alterae verborum)“ (Rhetorica ad Herennium 1998 [ca. 85 v. Chr.], 171). Weil solche Bilder ihre Gegenstände nicht abbilden, wird in ihnen „die Repräsentations- durch eine Erinnerungsfunktion“ überlagert (Lachmann 1990, 29): „Im Konzept des Fingierens der imagines oder simulacra [...] ist die Entähnlichung enthalten“ (ebd.).

\section{Detaillieren}

„Eine Sprachbildlichkeit im Sinne von Metaphorik kommt nicht durch Gegenstandsreferenz, sondern durch Autoreferenz zustande“, so fasst es Eckhard Lobsien zusammen (Lobsien 1990, 97). Von den Figuren der Vergegenwärtigung unterscheidet die Rhetorik deshalb die Figuren der Detaillierung (amplificatio) (vgl. Campe 1990, 5). Ihr „Texteffekt [...], der durch gezielte Strategien einer Rücknahme jener Selbstpräsentationstendenzen der Sprache erzeugt wird“, steht im Dienst einer „quasi-wahrnehmungsmäßige[n] Gegenstandserfassung“ (Lobsien 1990, 97). Detaillierungen basieren darauf, dass ein Gegenstand in seinen Eigenschaften erfasst, und das heißt umgekehrt, in seine Eigenschaften zerlegt wird. Weil Detaillierung mehr oder weniger ausdrücklich mit Wahrnehmung kalku- 
liert, sodass dieses bildgebende Verfahren in literarischen Texten nicht selten mit einer personalisierten Wahrnehmungssituation einhergeht (vgl. Mergenthaler 2002, 321-345), fallen in den entsprechenden Figuren Rhetorik und Ästhetik in eins. Indem Detaillierungen auf die - in einer Formulierung von Martin Seel „phänomenale Präsenz“ (Seel 2000, 52) der Gegenstände treffen, basieren sie auf „phänomenale[r] Individualität“ (ebd., 56). Seel veranschaulicht die ästhetische Wahrnehmung am Beispiel eines roten Balls, der seinem Nachbarsjungen Oskar gehört: Dieser Ball ist nicht nur rund, rot und aus Leder, sondern darüber hinaus hat er auch Eigenschaften, die von einer Person wie Oskar oder seiner Mutter sowohl von ihrem Standpunkt und ihrer Perspektive als auch von ihren Neigungen, Meinungen und Interessen - abhängen.

Die Rhetorik katalogisiert zwar sehr viele Figuren der Detaillierung, jedoch liegt strukturell allen die Matrix der Wiederholung (geminatio) zugrunde. Denn mit jeder Detaillierung wird die einstellige res-verbum-Relation verdoppelt: Mindestens zwei, tendenziell (unendlich) viele Wörter drücken eine Sache aus, sodass auch in der Detaillierung Semiosis über Mimesis triumphiert. Grundsätzlich lassen sich inner- von außerthematischen Detaillierungen unterscheiden: Erstere bestimmen nur einen Gegenstand, letztere überschreiten die Grenze von einem Gegenstand $\mathrm{zu}$ anderen. $\mathrm{Zu}$ dieser ersten Unterscheidung kommt eine zweite hinzu. Denn es gibt Detaillierungen, die einen Gegenstand in seine Eigenschaften gliedern, indem sie diese subordinieren. Sie bezwecken eine abgeschlossene beziehungsweise geschlossene Darstellung des Gegenstands, die ihn in sämtlichen Eigenschaften erfassen soll. Detaillierungen können jedoch auch die Eigenschaften eines Gegenstands sammeln und lediglich koordinieren, ohne den Gegenstand in seinen mannigfaltigen Eigenschaften und Beziehungen vollständig darzustellen (vgl. Lausberg 2008 [1960], 336-346). „Texte dieser Art“ können sowohl „anschaulicher als auch kryptischer sein als andere“ (Plett 1991 [1971], 45). In literarischen Texten gehen Detaillierung und Reihenbildung daher Hand in Hand - etwa wenn der Sänger im zweiten Gesang der Ilias anhebt: „Die Führer aber der Schiffe will ich nennen und die Schiffe allesamt“ (Homer 1977 [8./7. Jh. v. Chr.], 35), um sie im Folgenden aneinanderzureihen. Sabine Mainberger unterscheidet drei Reihungstypen: die endliche, erschöpfend darzustellende Reihe, die unendliche, nicht erschöpfend darzustellende Reihe sowie die endliche, aber empirisch kaum zu erfassende, deshalb nicht erschöpfend darzustellende Reihe (vgl. Mainberger 2003, 11). Geht man davon aus, dass es den ersten Typ nur idealiter geben kann, sind Detaillierungen realiter immer davon bedroht, ihren Gegenstand ,aus den Augen zu verlieren` beziehungsweise ihn buchstäblich zu zerreden.

Unter den Detaillierungen spielt die Beschreibung (descriptio) eine Hauptrolle für die literarische Bildlichkeit. In den ersten Sätzen seiner Erzählung Der 
Schatten des Körpers des Kutschers (1960) verbindet etwa Peter Weiss Beschreibung und Aufzählung (enumeratio): „Durch die halboffene Tür sehe ich den lehmigen, aufgestampften Weg und die morschen Bretter um den Schweinekofen. Der Rüssel des Schweins schnuppert in der breiten Fuge [sic!] wenn er nicht schnaufend und grunzend im Schlamm wühlt. Außerdem sehe ich noch ein Stück der Hauswand, mit zersprungenem, teilweise abgebröckeltem gelblichen Putz, ein paar Pfähle, mit Querstangen für die Wäscheleinen, und dahinter, bis zum Horizont, feuchte, schwarze Ackererde“ (Weiss 1991 [1960], 9). In dieser Beschreibung machen nicht nur die iterativ-durativen Zeitraffungen mithilfe der Verben, sondern vor allem auch die mit einer konzessiven, koordinierenden Konjunktion eingeleitete Negation in selbstreflexiver Wendung auf den referentiellen Fehlschluss (referential fallacy) der Detaillierung aufmerksam (vgl. Riffaterre 1978a). Tatsächlich kann der Ich-Erzähler nicht sehen, ob der Rüssel des Schweins ,in der breiten Fuge“ „schnuppert“ oder - ,x ist der Fall, wenn nicht y der Fall ist“ - „schnaufend und grunzend im Schlamm wühlt“ und selbstverständlich auch nicht, dass der Rüssel - Synekdoche des Schweins - schnauft und grunzt. Letzteres kann er möglicherweise hören, die gesamte Szene aber allenfalls erinnern. Dergestalt erweist sich die Beschreibung als eine radikal konstruktivistische Figur, die Wahrnehmungen, Erinnerung und vor allem Wissen akkumuliert, sodass sich die Beschreibung mit ,sozialer Energie‘ anreichert (vgl. Drügh 2006, 371-409). Deshalb wendet sich Emil Angehrn entschieden gegen die „Grundillusion [...] des Immediatismus des Beschreibens“ (Angehrn 1995, 65) - und zwar sowohl gegen den Immediatismus der Beschreibung im Allgemei-

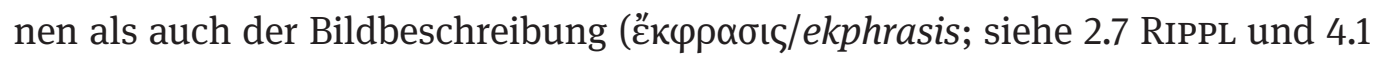
WANDHOFF) sowie Kunstbeschreibung im Besonderen: „Die Differenz zwischen Fiktion und Abbildrealismus“ ist lediglich „eine Frage der Konvention“ (ebd., 72). Denn ein Bild ihres Gegenstandes produziert die Beschreibung völlig unabhängig davon, ob sie auf Wirklichkeit referiert oder nicht. Deshalb kann Rilke in seinem ekphrastischen Sonett - um das Beispiel des Archaïschen Torsos Apollos noch einmal aufzugreifen - durch ein bildgebendes Verfahren, das Vergegenwärtigung mit Detaillierung verbindet, dem Gott ein Gesicht geben. Mittels der Gedanken-

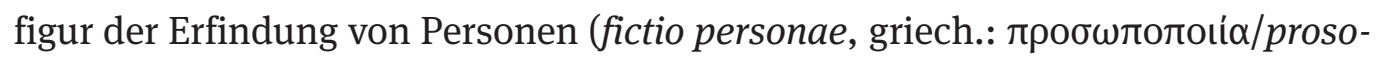
popoiia) ertönt im letzten Vers in selbstreferentieller Wendung die Stimme Apollons als Stimme des Gedichts (vgl. Menke 2000, 137-216). Seine Botschaft variiert die Inschrift am Eingang des Delphischen Tempels ,Erkenne dich selbst“ ( $y v \tilde{\omega} \theta \mathrm{u}$

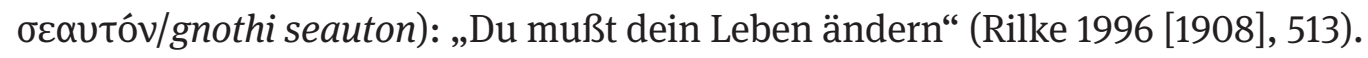




\section{Erzählen}

Das Problem der Anschaulichkeit führt in der Rhetorik also zum einen zur Stillehre und dort zu den Figuren der Vergegenwärtigung und Detaillierung, zum anderen zur Gedächtnislehre und dort zu den imagines agentes. Darüber hinaus spielt Anschaulichkeit aber auch im ersten Teil des rhetorischen Systems, der (Er-)Findungslehre, und dort bei der Einteilung der Rede unter dem Stichwort ,Erzählung“ (narratio) eine wichtige Rolle (vgl. Solbach 1994, 75-97): „Anschaulichkeit (evidentia) ist zwar [...] in der Erzählung ein großer Vorzug, indem etwas Wahres nicht nur ausgesprochen (dicere), sondern gewissermaßen vorgeführt zu werden verdient (ostendere), doch kann man sie zur Deutlichkeit (perspicuitas) rechnen“ (Quintilian 1995a [ca. 95 n. Chr.], 461 und 463). Mit dieser Wendung verweist Quintilian zur weiteren Klärung des Problems auf die Gedankenfiguren

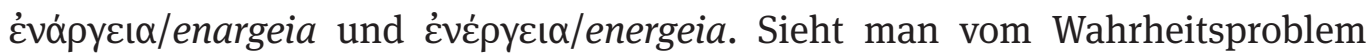
einmal ab, geht es Quintilian auch hier um den Unterschied von propositionalem Sagen und theatralem Zeigen mit belehrender, aber ohne emotionale Wirkung. Gedankenfiguren und narratives Genre verweisen innerhalb des rhetorischen Systems also wechselseitig aufeinander, wobei beider Performativität den Drehund Angelpunkt der Beziehung bildet.

Tatsächlich verhandelt Quintilian die narratio aber nicht nur als Abschnitt der Rede (pars orationis), sondern auch als „gerahmte[] Stelle“ (Campe 1997, 219), die den Stellenwert einer Figur oder eines Tropus hat. Um die Wirkung einer solchen ,Stelle‘ zu erklären, greift er in der Stillehre auf den Tropus der Allegorie zurück - den Narrationstropus. „[A]nders als auf dem öffentlichen Marktplatz, der Agora, reden“ (Kurz 2004 [1976], 34), so lautet die wörtliche Übersetzung der

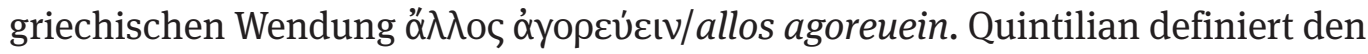
Tropus in diesem Sinn als metaphora continua: „Die Allegorie, die man im Lateinischen als inversio (Umkehrung) bezeichnet, stellt einen Wortlaut dar, der entweder einen anderen oder gar zuweilen den entgegengesetzten Sinn hat [Ironie, d. Verf.]. Die erste Art erfolgt meist in durchgeführten Metaphern“ (Quintilian 1995b [ca. 95 n. Chr.], 237). Sein Beispiel findet er bei Horaz: „So etwa, ,Schiff, dich treibt die Flut wieder ins Meer zurück! | Weh, was tust du nur jetzt! Tapfer dem Hafen $\mathrm{zu}^{\prime}$ und die ganze Stelle bei Horaz, an der er Schiff für das Gemeinwesen, Fluten und Stürme für Bürgerkriege, Hafen für Frieden und Eintracht sagt“ (ebd.).

Während sich die Allegorieforschung vorwiegend um das Verhältnis von wörtlicher (sensus litteralis) und allegorischer Bedeutung (sensus allegoricus) in vollständigen (allegoria tota) oder ausgelegten Allegorien (allegoria permixta) gekümmert hat, ist die narrative Struktur des Tropus ins Hintertreffen geraten (vgl. Alt 1995, 3-33; Drügh 2000, 7-30). Weil diese Narrativität aber bereits in der Sukzessivität der Veranschaulichung angelegt ist, verlangt sie gewisserma- 
ßen nach einem eigenen Tropus. Dabei kennt Quintilian ebenso Allegorien mit Wörtern, die in übertragener, uneigentlicher Bedeutung verwendet werden (allegoria cum translationibus), wie solche mit Wörtern, die in eigentlicher Bedeutung verwendet werden (allegoria sine translationibus); was sie verbindet, ist ihre Zeitlichkeit. „The problem of allegorical narrative [is] primarily a temporal issue“ (Fineman 1981, 26), so bringt es Joel Fineman in Bezug auf Roman Jakobsons strukturalistisches Sprachmodell auf den Punkt. „[A]llegory would be the poetical projection of the metaphoric axis onto the metonymic“ (ebd., 31) - und zwar unter folgender Voraussetzung: „[M]etaphor is understood as the synchronic system of difference, which constitutes the order of language (langue), and metonymy as the diachronic principle of combination and connection by means of which structure is actualized in time in speech (parole)“ (ebd.).

Eine Sonderform der Allegorie ist die Ersetzung von Abstrakta durch handelnde und sprechende Personen mittels der bereits erwähnten Gedankenfigur der Prosopopoiia, die im Hinblick auf die in der Anrede erzeugte Performativität des Quintilian-Beispiels („Schiff, dich treibt ...“) interessant ist. Durch diese Verlebendigung gewinnt die Allegorie nämlich an Anschaulichkeit. Während allegorische Personifikationen normalerweise intradiegetisch auftauchen, figu-


das intradiegetische Schiff eine extradiegetische Stimme in der zweiten Person Singular, die den Erzählakt durch den Ausruf (exclamatio) inszeniert, ja theatralisiert: „Weh was tust Du nur jetzt!“ (Quintilian 1995b [ca. 95 n. Chr.], 237) Dergestalt implementiert er der Allegorie also eine Vermittlungsinstanz, während die Verben - zurücktreiben, treiben und (zu-)bewegen - den Tropus verzeitlichen, sodass Erzählzeit und erzählte Zeit in einer doppelten zeitlichen Sequenz auseinandertreten. Allegorie und Erzählung fallen also in dieser narrativen Struktur zusammen.

Daraus folgt, dass Erzählungen in der ersten Person Singular besonders anschaulich, weil besonders lebendig sind. Denn in der Regel ist der narrative Akt in solchen Erzählungen sowohl körper- und perspektivgebunden als auch kommunikations- und traditionsbezogen. Man kann sich also die Figur vorstellen, die wie beispielsweise in Franz Kafkas Erzählung Die Brücke (1917) ihre eigene Geschichte erzählt: „Ich war steif und kalt, ich war eine Brücke, über einem Abgrund lag ich. Diesseits waren die Fußspitzen, jenseits die Hände eingebohrt, in bröckelndem Lehm habe ich mich festgebissen. Die Schöße meines Rockes wehten zu meinen Seiten. In der Tiefe lärmte der eisige Forellenbach“ (Kafka 1993 [1917], 304). Die Anschaulichkeit der Erzählung basiert auf der Fokalisierung beziehungsweise Konzeptualisierung durch die in erster Person Singular (intradiegetisch-autodiegetisch) erzählende Brücke, genauer gesagt von der Verkörperung (embodiment) dieses Erzählens, zum Beispiel der Position der Hände, 
der Füße sowie der Wahrnehmung der Brücke (vgl. Herman 2002, 301-330). Die Anschaulichkeit nimmt ab, sobald stattdessen eine logisch außerhalb der fiktiven Welt angesiedelte Instanz in dritter Person (extradiegetisch-heterodiegetisch) über die Brücke erzählt, bis die Erzählsituation von dieser Rahmen- wieder in die Binnenerzählung wechselt: „Kein Tourist verirrte sich zu dieser unwegsamen Höhe, die Brücke war in den Karten noch nicht eingezeichnet. - So lag ich und wartete; ich mußte warten. Ohne einzustürzen kann keine einmal errichtete Brücke aufhören, Brücke zu sein“ (Kafka 1993 [1917], 304).

Das Leistungsprofil des Referenzmediums ,Bild‘, an dem sich das Funktionsund Leistungsprofil des literarischen Textes bemisst, wenn ihm Bildlichkeit zugeschrieben wird, gerät mit der narrativen Allegorie freilich an seine Grenzen. Erst am Ende des 19. Jahrhunderts holen die visuellen Medien die literarischen Texte wieder ein, die im Spannungsfeld von Narrativität und Performativität bewegte Bilder erzeugen. Denn ,filmisch` verfährt die Allegorie, lange bevor der Film als bewusstseins-, wahrnehmungs- und erfahrungsprägendes Leitmedium für die Besetzung der Unbekannten in der Funktion ,Bildlichkeit‘ dem Bild den Rang abläuft (siehe 4.10 HARRIS und 4.11 TRIPP); das zeigt beispielhaft die protokinematografische Wahrnehmung einer Eisenbahnfahrt in Julius Harts Auf der Fahrt nach Berlin (1885), in dem die Landschaftsbilder vor dem inneren Auge des Rezipienten vorbeifliegen: „[...] Zerzauste Wolken, winddurchwühlter Wald | Und braune Felsen schießen wirr vorüber, | Dort graut die Havel, und das Wasser schwallt, | Die Brücke, hei! dumpf braust der Zug hinüber.“ (Hart 1982 [1885], 42) In der Kombination von Figuren der Detaillierung, Narrativierung und Performativität („hei!“) erzeugt das Gedicht kein statisches Gesamtbild, sondern eine dynamische Bildfolge, die als Allegorie des Fortschritts darüber hinaus die literarische Bildlichkeit in den Dienst semantischer Komplexität stellt.

\section{Textbildlichkeit}

Für das Funktions- und Leistungsprofil des literarischen Textes, anschauliche Vorstellungen zu erzeugen, gibt die Rhetorik Begriffe an die Hand, die bis heute an Aktualität nichts verloren haben. Doch im Gegensatz zu späteren Literaturtheorien beschränkt die Rhetorik ihr Interesse an der Bildlichkeit keineswegs auf Figuren und Tropen. Stattdessen kalkuliert diese Texttheorie sehr differenziert mit den beiden Modalitäten ,Sprache‘ und ,Bild‘. Neben der gesprochenen Rede berücksichtigt die Rhetorik daher auch den geschriebenen Text. Immer wieder finden sich in den Lehrbüchern Hinweise auf die Schrift, die nicht nur als (sekundäres) Zeichensystem der Rede, sondern auch in ihrer eigenen ,Schriftbildlich- 
keit' beachtet wird (vgl. 2.1 KAMMER). So sind eine Reihe von Figuren rein grafisch bestimmt, wie das von Cicero 44 v. Chr. in De Divinatione (,Über die Weissagung`) angeführte Akrostichon „Q. Ennius fecit“ („Q. Ennius ist der Verfasser“), das die Anfangsbuchstaben eines Gedichts des Quintus Ennius bildet (Cicero 1991 [44 v. Chr.], 238). Ebenso wie die antiken Rhetoriker kennt auch Alexander Gottlieb Baumgarten, der aus der Rhetorik eine literarische Medienästhetik entwickelt, das Buchstabenspiel (ludus litterarum). Darüber hinaus führt er in seinen Meditationes philosophicae de nonnullis ad poema pertinentibus (1735) in der Tradition der frühneuzeitlichen Rhetorik Gestalt-Figuren (expressiones figurarum) ein (vgl. Baumgarten 1983 [1735], 78-79), die man heute unter dem Oberbegriff der visuellen Poesie zusammenfasst (vgl. Berndt 2011, 75; Ernst und Adler 1987, 73-86). Mit ihnen steht freilich mehr als die ,Schriftbildlichkeit‘ zur Diskussion. Baumgarten entdeckt vielmehr eine genuine ,Textbildlichkeit‘ (vgl. Giuriato und Kammer 2006, 7-24). Literarische Texte, die sich durch eine solche Eigenschaft auszeichnen, sind wie Bilder selbst Gegenstände der Wahrnehmung, weil sie sowohl gelesen als auch gesehen werden wollen (vgl. 4.14 WEINGART).

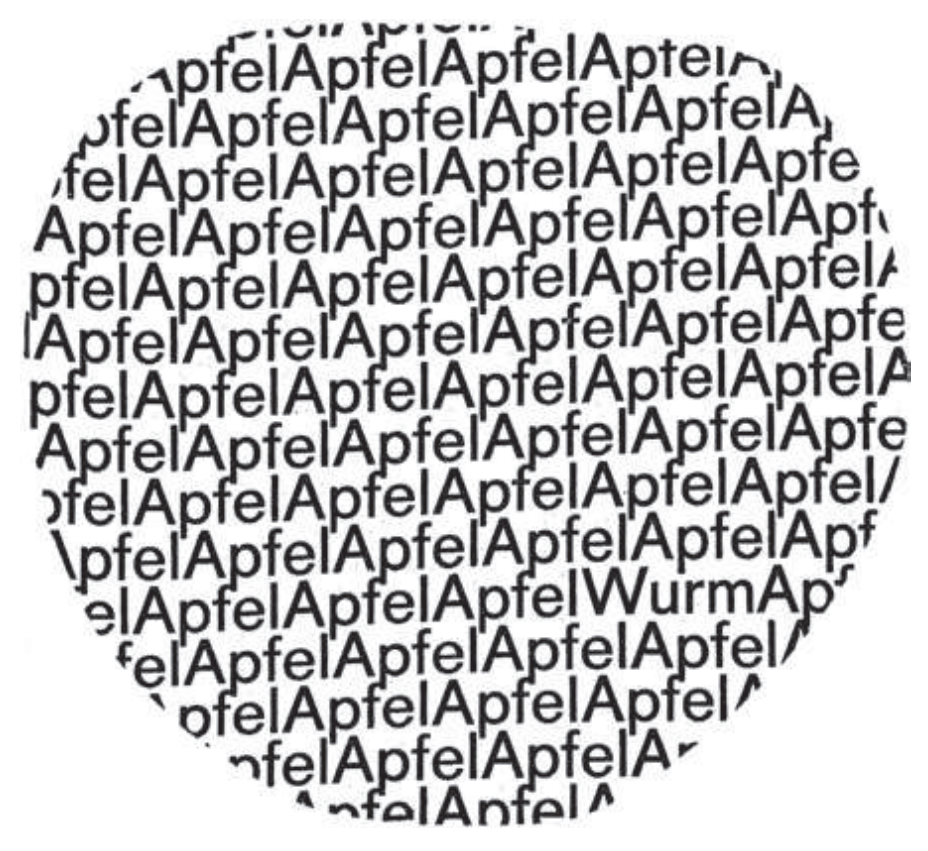

Abb. 3: Reinhard Döhl: Apfel, Postkarte, 1965, Stuttgart

Ein bekanntes Beispiel für ein dergestalt wahrnehmungsnahes Textbild stellt Reinhard Döhls als sogenannte ,Konkrete Poesie‘ entstandener Apfel (1965) dar (Abb. 3). Dieses Beispiel zeigt, dass Bildlichkeit in einem literarischen Text gleichzeitig mit sprachlichen und bildlichen Mitteln realisiert werden kann. Der Text basiert nämlich auf der Figur der Wiederholung, die das Wort „Apfel“ vervielfäl- 
tigt. Schon durch die fehlende Worttrennung (scriptura continua) widersetzt sich der Apfel dem ,Gelesen-Werden'. Zum ,Gesehen-Werden' fordert der Text dadurch auf, dass er die Form eines Apfels abbildet. Beim ,Sehen' wiederum springt nun ein grafisches Element besonders ins Auge: In der rechten unteren Mitte des Apfels befindet sich das Wort ,Wurm‘. Dieser nimmt jedoch nicht visuell Gestalt an, sondern dadurch, dass das im Lexikon abgelegte Wort „Wurm“ eine anschauliche Vorstellung erzeugt. Im wahrgenommenen Apfel ist also der vorgestellte Wurm ,drin‘. Durch dieses Zusammenspiel von ,Sehen' und ,Lesen“ entsteht ein Medienhybrid, dessen Bildlichkeit sowohl sprachliche Zeichen (,Sprachbildlichkeit') als auch bildliche Zeichen (,Textbildlichkeit‘) erzeugen. Seine semantische Komplexität erhält der Wurm, der im Apfel ,drin‘ ist, dabei allerdings erst im Horizont des kulturellen Gedächtnisses durch die mannigfaltig vermittelte Ähnlichkeit mit demjenigen Wurm, der in der ganzen Schöpfung ,drin' ist. War es nicht die Schlange, die Eva verführt hat, Adam zu verführen, den Apfel vom Baum der Erkenntnis zu pflücken? Doch würde man die Multimodalität des Textes nicht berücksichtigen, könnte der Apfel nicht die volle Bedeutung entfalten, die genau diesen Medienhybrid zum witzigen Symbol des Sündenfalls macht.

\section{Literaturverzeichnis}

Alt, Peter-André. Begriffsbilder. Studien zur literarischen Allegorie zwischen Opitz und Schiller. Tübingen: Niemeyer, 1995.

Angehrn, Emil. „Beschreibung zwischen Abbild und Schöpfung“. Beschreibungskunst Kunstbeschreibung. Ekphrasis von der Antike bis zur Gegenwart. Hrsg. von Gottfried Boehm und Helmut Pfotenhauer. München: Fink, 1995. 59-74.

Aristoteles. Werke in deutscher Übersetzung Bd. 4: Rhetorik. Übers. und erl. von Christof Rapp. Hrsg. von Hellmut Flashar. Berlin: Akademie-Verlag, 2002 [ca. 349-336 v. Chr.].

Aristoteles. Werke in deutscher Übersetzung Bd. 5: Poetik. Übers. und erl. von Arbogast Schmitt. Hrsg. von Hellmut Flashar. 2., durchges. und ergänzte Aufl. Berlin: Akademie-Verlag, 2011 [ca. 335 v. Chr.].

Baumgarten, Alexander Gottlieb. Meditationes philosophicae de nonnullis ad poema pertinentibus/Philosophische Betrachtungen über einige Bedingungen des Gedichtes. Übers. und hrsg. von Heinz Paetzold. Neudruck des lateinischen Textes von 1936. Hamburg: Meiner, 1983 [1735].

Berndt, Frauke. „Topik-Forschung“. Gedächtniskonzepte der Literaturwissenschaft. Theoretische Grundlegung und Anwendungsperspektiven. Hrsg. von Astrid Erll und Ansgar Nünning. Berlin und New York, NY: De Gruyter, 2004. 31-52.

Berndt, Frauke. Poema/Gedicht. Die epistemische Konfiguration der Literatur um 1750. Berlin und Boston, MA: De Gruyter, 2011.

Campe, Rüdiger. Affekt und Ausdruck. Zur Umwandlung der literarischen Rede im 17. und 18. Jahrhundert. Tübingen: Niemeyer, 1990. 
Campe, Rüdiger. „Vor Augen stellen. Über den Rahmen rhetorischer Bildgebung“.

Poststrukturalismus. Herausforderung an die Literaturwissenschaft. DFG-Symposion 1995.

Hrsg. von Gerhard Neumann. Stuttgart und Weimar: Metzler, 1997. 208-225.

Cicero, Marcus Tullius. Über die Wahrsagung/De Divinatione. Lateinisch/deutsch. Übers., hrsg. und erl. von Christoph Schäublin. Darmstadt: Wissenschaftliche Buchgesellschaft, 1991 [44 v. Chr.].

Cicero, Marcus Tullius. Orator. Lateinisch/deutsch. Hrsg. und übers. von Bernhard Kytzler. 4., durchges. Aufl. Düsseldorf und Zürich: Artemis \& Winkler, 1998 [46 v. Chr.].

Cicero, Marcus Tullius. De Oratore/Über den Redner. Lateinisch/deutsch. Übers. und hrsg. von Theodor Nüßlein. Düsseldorf und Zürich: Artemis \& Winkler, 2007 [55 v. Chr.].

Derrida, Jacques. „Die weiße Mythologie. Die Metapher im philosophischen Text“ [1972]. Randgänge der Philosophie. Übers. von Gerhard Ahrens. Hrsg. von Peter Engelmann. Wien: Passagen, 1988. 205-258.

Döhl, Reinhard. Apfel. Postkarte. Stuttgart, 1965.

Drügh, Heinz J. Anders-Rede. Zur Struktur und historischen Systematik des Allegorischen. Freiburg im Breisgau: Rombach, 2000.

Drügh, Heinz J. Ästhetik der Beschreibung. Poetische und kulturelle Energie deskriptiver Texte (1700-2000). Tübingen: Francke, 2006.

Ernst, Ulrich, und Jeremy Adler. Text als Figur. Visuelle Poesie von der Antike bis zur Moderne. Weinheim: VCH, 1987.

Fauth, Wolfgang. „Apollon“. Der kleine Pauly. Lexikon der Antike Bd. 1: Aachen bis Dichalkon. Hrsg. von Konrat Zeigler und Walther Sontheimer. Stuttgart: Druckenmüller, 1964. 441-448.

Fineman, Joel. „The Structure of Allegorical Desire“. Allegory and Representation. Hrsg. von Stephen J. Greenblatt. Baltimore, MD, und London: Johns Hopkins University Press, 1981. 26-59.

Giuriato, Davide, und Stephan Kammer (Hrsg.). Bilder der Handschrift. Die graphische Dimension der Literatur. Frankfurt am Main und Basel: Stroemfeld, 2006.

Groddeck, Wolfram. Reden über Rhetorik. Zu einer Stilistik des Lesens. 2., durchges. Aufl. Frankfurt am Main und Basel: Stroemfeld, 2008 [1995].

Hart, Julius. „Auf der Fahrt nach Berlin“ [1885]. Lyrik des Naturalismus. Hrsg. von Jürgen Schulte. Stuttgart: Reclam, 1982. 42-44.

Herman, David. Story Logic. Problems and Possibilities of Narrative. Lincoln, NE: University of Nebraska Press, 2002.

Homer. Ilias. Übers. von Wolfgang Schadewaldt. 2. Aufl. Frankfurt am Main: Insel, 1977 [8/7. Jh. v. Chr.].

Horaz [Quintus Horatius Flaccus]. „De arte poetica liber/Das Buch von der Dichtkunst“ [14 v. Chr.]. Sämtliche Werke. Lateinisch und Deutsch. Teil I nach Kayser, Nordenflycht, Burger hrsg. von Hans Färber, Teil II übers. und bearb. von Hans Färber und Wilhelm Schöne. Darmstadt: Wissenschaftliche Buchgesellschaft, 1967. 230-259.

Kafka, Franz. „Die Brücke“ [1917]. Schriften, Tagebücher, Briefe. Kritische Ausgabe Bd. 4.1: Nachgelassene Schriften und Fragmente I. Hrsg. von Malcolm Pasley. Frankfurt am Main: Fischer, 1993. 304-305.

Kohl, Katrin. Metapher. Stuttgart und Weimar: Metzler, 2007.

Kurz, Gerhard. Metapher, Allegorie, Symbol. 5., durchges. Aufl. Göttingen: Vandenhoeck \& Ruprecht, 2004 [1976]. 
Lachmann, Renate. Gedächtnis und Literatur. Intertextualität in der russischen Moderne. Frankfurt am Main: Suhrkamp, 1990.

Lausberg, Heinrich. Handbuch der literarischen Rhetorik. Eine Grundlegung der Literaturwissenschaft. 4. Aufl. Stuttgart: Steiner, 2008 [1960].

Lobsien, Eckhard. „Bildlichkeit, Imagination, Wissen: Zur Phänomenologie der Vorstellungsbildung in literarischen Texten“. Bildlichkeit. Internationale Beiträge zur Poetik. Hrsg. von Volker Bohn. Frankfurt am Main: Suhrkamp, 1990. 89-114.

Mainberger, Sabine. Die Kunst des Aufzählens. Elemente zu einer Poetik des Enumerativen. Berlin und New York, NY: De Gruyter, 2003.

Mauthner, Fritz. Beiträge zu einer Kritik der Sprache Bd. 2: Zur Sprachwissenschaft. Reprografischer Nachdruck der Ausgabe Leipzig 1923. Hildesheim: Olms, 1967 [1912].

Menke, Bettine. Prosopopoiia. Stimme und Text bei Brentano, Hoffmann, Kleist und Kafka. München: Fink, 2000.

Mergenthaler, Volker. Sehen schreiben - schreiben sehen. Literatur und visuelle Wahrnehmung im Zusammenspiel. Tübingen: Niemeyer, 2002.

Plett, Heinrich F. Einführung in die rhetorische Textanalyse. 8. Aufl. Hamburg: Buske, 1991 [1971].

Quintilian [Marcus Fabius Quintilianus]. Ausbildung des Redners. Teil 1: Buch I-VI. Übers. und hrsg. von Helmut Rahn. 3. Aufl. Darmstadt: Wissenschaftliche Buchgesellschaft, 1995a [ca. 95 n. Chr.].

Quintilian [Marcus Fabius Quintilianus]. Ausbildung des Redners. Teil 2: Buch VII-XII. Übers. und hrsg. von Helmut Rahn. 3. Aufl. Darmstadt: Wissenschaftliche Buchgesellschaft, 1995b [ca. 95 n. Chr.].

Rhetorica ad Herennium. Lateinisch/deutsch. Übers. und hrsg. von Theodor Nüßlein. 2. Aufl. Düsseldorf und Zürich: Artemis \& Winkler, 1998 [ca. 85 v. Chr.].

Ricœur, Paul. Die lebendige Metapher. Übers. von Rainer Rochlitz. München: Fink, 1986 [1975].

Riffaterre, Michael. „Referential Fallacy“. Columbia Review 57.2 (1978a): 21-35.

Riffaterre, Michael. Semiotics of Poetry. Bloomington, IN: Indiana University Press, 1978b.

Rilke, Rainer Maria. „Archaïscher Torso Apollos“ [1908]. Werke. Kommentierte Ausgabe in vier Bänden Bd. 1: Gedichte 1895 bis 1910. Hrsg. von Manfred Engel und Ulrich Fülleborn. Frankfurt am Main: Insel, 1996. 513.

Sachs-Hombach, Klaus. Das Bild als kommunikatives Medium. Elemente einer allgemeinen Bildwissenschaft. 3., verbesserte Aufl. Köln: Halem, 2013 [2003].

Seel, Martin. Ästhetik des Erscheinens. München: Hanser, 2000.

Solbach, Andreas. Evidentia und Erzähltheorie. Die Rhetorik anschaulichen Erzählens in der Frühmoderne und ihre antiken Quellen. München: Fink, 1994.

Starobinski, Jean. Wörter unter Wörtern. Die Anagramme von Ferdinand de Saussure. Übers. von Henriette Beese. Frankfurt am Main, Berlin und Wien: Ullstein, 1980.

Tonger-Erk, Lily. Actio. Körper und Geschlecht in der Rhetoriklehre. Berlin und Boston, MA: De Gruyter, 2012.

Weiss, Peter. „Der Schatten des Körpers des Kutschers“ [1960]. Werke Bd. 2: Prosa II. Der Schatten des Körpers des Kutschers. Abschied von den Eltern. Fluchtpunkt. Das Gespräch der drei Gehenden. Rekonvaleszenz. Hrsg. vom Suhrkamp Verlag in Zusammenarbeit mit Gunilla Palmstierna-Weiss. Frankfurt am Main: Suhrkamp, 1991. 7-56.

Willems, Gottfried. Anschaulichkeit. Zu Theorie und Geschichte der Wort-Bild-Beziehungen und des literarischen Darstellungsstils. Tübingen: Niemeyer, 1989. 
Willer, Stefan. „Orte, Örter, Wörter. Zum locus ab etymologia zwischen Cicero und Derrida“. Rhetorik. Figuration und Performanz. DFG-Symposion 2002. Hrsg. von Jürgen Fohrmann. Stuttgart und Weimar: Metzler, 2004. 39-58. 